\title{
Desafíos para la Construcción de Políticas Educacionales de los Gobiernos Subnacionales chilenos: El caso de la Región del Maule ${ }^{1}$
}

\author{
Desafios para a Construção da Política de Educação Governos \\ subnacionais chilenos:o caso da Região Maule
}

\author{
Challenges for Building the Educational Policy Chilean \\ subnational governments: The case of the Maule Region
}

\section{Giselle Davis-Toledo² Sebastián Donoso-Díaz ${ }^{3}$}

\section{Resumen}

El artículo analiza las prácticas de toma de decisiones en los gobiernos subnacionales en Chile, país con una fuerte tradición centralista, revisando las lógicas seguidas y los procesos implementados, con especial referencia a las políticas educativas regionales. Se examinan las tensiones entre los diversos niveles de gestión del gobierno central y subcentral, y el uso de los instrumentos regionales de planificación para la elaboración de propuestas educativas a nivel subnacional. Desde el punto de vista metodológico, se emplea el enfoque de la Teoría Fundamentada, la cual orienta el análisis cualitativo de un corpus empírico de 73 entrevistas a autoridades regionales. Los principales resultados dan cuenta que tanto el gobierno subnacional como los partidos políticos no cuentan con proyectos educativos con arraigo territorial. Las políticas de descentralización han sido más bien producto de pactos electorales que fruto de un convencimiento de visiones de ciudadanía y de desarrollo socioeconómico, respondiendo a la dinámica de los procesos nacionales. La precariedad de la descentralización y la dominancia de las prácticas de reproducción del poder de los partidos políticos obstaculizan la elaboración de proyectos de desarrollo regional. Se concluye que el proyecto educativo regional requiere un cambio cultural en las prácticas de gobernanza de los gobiernos subnacionales, cuestión transversal al desarrollo de la sociedad civil en estos entornos territoriales.

Palabras claves: Descentralización; Educación;Prácticas sociales; Toma de decisiones;Gobernanza.

1El artículo es parte de los Proyectos PIA CONICYT PSD 60; PIA CONICYT CIAE 05.

2 Doctora en Ciencias Sociales aplicadas y académica del Departamento de Salud Pública de la Universidad de Talca (Chile), gdavis@utalca.cl 3 Doctor en Educación y académico del Instituto de Investigación y Desarrollo Educacional de la Universidad de Talca (Chile), sdonoso@utalca.cl. 


\title{
Resumo
}

O artigo analisa as práticas de tomada de decisões nos governos subnacionais do Chile, país com uma forte tradição centralizadora, revisando as lógicas seguidas e os processos implementados, com especial referência às políticas educativas regionais. São examinadas as tensões entre os diversos níveis de gestão do governo central e subcentral, e o uso dos instrumentos regionais de planejamento para a elaboração de propostas educativas em nível subnacional. Do ponto de vista metodológico, emprega-se o enfoque da Teoria Fundamentada, a qual orienta a análise qualitativa de um corpo empírico de 73 entrevistas com autoridades regionais. Os principais resultados dão conta que tanto o governo subnacional como os partidos políticos não contam com projetos educacionais com relações territoriais. As políticas de descentralização têm sido mais produto de pactos eleitorais do que fruto de um convencimento de visões de cidadania e de desenvolvimento socioeconômico, respondendo à dinâmica dos processos nacionais. A precariedade da descentralização e a dominância das práticas de reprodução do poder dos partidos políticos obstacularizam a elaboração de projetos de desenvolvimento regional. Conclui-se que o projeto educacional regional requer uma mudança cultural nas práticas de governança dos governos subnacionais, questão transversal ao desenvolvimento da sociedade civil nestes entornos territoriais.

Palavras-chave: Descentralização; Educação; Práticas sociais; Tomada de decisões; Governança.

\begin{abstract}
The article explores the decision making processes of Chile's subnational governments, a country with a strong centralist tradition, reviewing the processes implemented, with a particular emphasis on regional education policies, a key part of descentralization, and their instrumental impact in territorial development strategies. This paper examines the tension between the different management levels of central and subcentralgovernment, and also the use of regional planning instruments to prepare educational projects of subnational level. From a methodological point of view, the grounded theory is used, the qualitative analysis of 73 interviews with regional and local authorities. The main result shows that both the central government and the political parties hace developed educational projects with strong territorial. Decentralization policies have been the result of electoral bargaining, following nation-wide trends which have been impulsed by socio-economic development and strongly held visions of citizenship. The precariousness of decentralization and the hegemony of political parties obstruct the creation of regional development policies. It is essential to promote the training and empowerment of human resources at local and regional government level to achieve efficient regionalization. The article concludes that regional education policy requires a cultural change in the governance of subnational authorities, which is essential for the development of civil society in these regions.
\end{abstract}

Key words: Decentralization; Education;Social practices; Decision making;Governance. 


\section{Presentación}

El Estado chileno en la actualidad da cuenta de las tensiones entre la tradición centralista con las renacientes políticas descentralizadoras que - tímidamente - han buscado un mayor equilibrio entre visiones más extremas. Bajo esta afirmación se analiza el desarrollo político alcanzado por el gobierno subnacional en la toma de decisiones de política educacional nacional, conjugados con las orientaciones esenciales de políticas subcentrales instaladas en los gobiernos regionales (GORE) del país, basados el caso del gobierno regional del Maule $^{4}$, centrándonos en el ámbito educacional, por su creciente importancia en el desarrollo territorial, la cual implica interacción efectiva a cada nivel de gobierno y de evidencia de su gobernanza en este nivel.

La relevancia de la educación es compartida por los gobiernos del continente que apuestan crecientemente a que el progreso dependerá en parte sustantiva de este factor como herramienta determinante, aunque no siempre exista plena consistencia entre esos anhelos, los objetivos y las formas como los mismos gobiernos subnacionales dan cuenta del aporte de las propuestas educativas que conforman su territorio en referencia al objetivo trazado.

Las políticas de educación pública en Chile son un elemento clave para la comprensión del desarrollo político de los gobiernos subnacionales, pues básicamente competen al gobierno central, el cual se reserva un conjunto de atribuciones consistentes con esta característica dominante de su hacer, en tanto se establecen y delegan otras funciones y tareas para las instancias subnacionales.

En razón de ello, se revisan los procesos de toma de decisiones de los gobiernos subnacionales en referencia al diseño e implementación de las políticas educacionales y/o del proyecto educacional que impulsan. El estudio de caso de la Región del Maule, responde a los vínculos territoriales que posee el equipo de investigación, a que se trata de una región central que no tiene marcadas características identitarias propias de las regiones de los extremos norte y sur, a saber: mayor tradición de autonomía, ni la fuerte identificación étnica de la Araucanía; como tampoco integra las regiones con mayor desarrollo económico/productivo, ni está directamente bajo el halo de influencia de la macro región Metropolitana. Es decir se trata de una región con menos factores explicativos de carácter contextual que algunos de los restantes macroterritorios del país.

La educación cumple dos funciones sociales claves para toda sociedad: la formación de capital humano inicial y avanzado - para el desempeño laboral en el más amplio sentido del término, y la formación ciudadana, es decir: la socialización de la población en aquellos valores y pautas de comportamiento para que sus ciudadanos funcionen en sociedad. Ambas cuestiones son políticamente relevantes para la gobernanza, por ello su apropiación y liderazgo dan cuenta del desarrollo político alcanzado por la instancia de gobierno. Desde esta perspectiva, el desarrollo de una política educacional subcentral, evidencia la realización del ideal modernizador en referencia de ambas funciones, implicando que los gobiernos subcentrales cuenten con un proyecto cohesionador de los actores e ideales sociales, del cual se generan políticas (educacionales) que orienten la toma de decisiones de inversión y que actúen sinérgicamente sobre el desarrollo de la región. Del logro de este objetivo depende la correcta orientación de las políticas de desarrollo, y en su dimensión más restrictiva, la calidad de la educación ofrecida.

Estudios importantes han demostrado que la coherencia política o calidad de la gobernanza municipal (local) se asocia a mejores resultados educativos (PAVÉZ, 2004; POLITEIA, 2008). En esa misma línea la OCDE plantea que "Ios Gobiernos Subnacionales o subcentrales cuenten con propuestas territoriales comunes, articuladas y multisectoriales que mejoren y proyecten la competitividad regional" (2009:35). El alcance y impacto de los proyectos educacionales obedecería a su vez, al grado en que integran las características y demandas de los sistemas educativos locales en las soluciones que proponen. Esto es, de su capacidad para generar una política

4 Localizada en el valle central de Chile, su capital regional es Talca. 
educativa local convocante, que signifique un posicionamiento regional y nacional de un proyecto de desarrollo (BOISIER, 2005:46; CARO, 2005). Al tenor de lo cual resulta importante reforzar el consenso entre los actores regionales sobre los objetivos, sus alcances, el rol y los medios disponibles para asegurar políticas territoriales de calidad (OCDE, 2009:41).

En torno de estos aspectos se analiza la relación entre gobernanza, descentralización y prácticas (acciones) en la toma de decisiones, desde los proyectos de desarrollo educacional en el gobierno regional del Maule; analizándose las acciones y visiones asociadas al diseño e implementación de las decisiones en este sector a nivel comunal y regional en el gobierno subnacional. El trabajo profundiza en las dinámicas de reproducción del poder político del Gobierno Subnacional en las prácticas decisionales y las posibilidades de construcción de un proyecto educacional en el horizonte del desarrollo regional. Complementariamente, se presenta una síntesis de las tendencias en el imaginario de los actores sobre ese horizonte; discutiéndose, finalmente, la importancia de revalorar el debate ideológico para el mejoramiento de la calidad de la educación en el país.

Teóricamente se asume el Gobierno Subnacional como un campo de relaciones recurrentes que reproducen el orden social y simultáneamente, producto de la reinterpretación que realizan los sujetos de las posibilidades en el contexto en que actúan, se redinamizan las relaciones (BOURDIEU, 2001; ZEMELMAN, 1992). Se entiende que las políticas de mejoramiento educativo no actúan de manera abstracta, ni se corresponden, necesariamente, con los supuestos utilizados en el nivel central para su diseño, sino que se insertan en realidades en las cuales los actores negocian, adaptan y redefinen su pertinencia empleando diversos procedimientos para ellos.

El problema de estudio se enfrenta desde un enfoque cualitativo de la gobernanza, con aplicación al campo educacional: interés reciente en la literatura, especialmente en lo referido a este sector, ya que poco se conoce en Chile sobre las visiones y reacciones de los sujetos frente a las políticas educativas, y menos respecto de la articulación entre las prácticas de toma de decisiones y los instrumentos de planificación, como por ejemplo, la Estrategias de Desarrollo Regional. Ello se explica porque la modernización del Estado chileno, basada en la eficiencia de los instrumentos de planificación, asume que la política implícita y/o explicita, es consensuada y suficiente para orientar efectivamente las acciones de desarrollo (COX, 2003).

Los estudios sobre gobernanza local son congruentes con los enfoques modernizadores del desarrollo que analizan la calidad de los sistemas educativos en función de su eficacia y eficiencia económica (MARCEL; RACZYNSKI, 2009). Paralelamente, procesos sociales complejos confluyen en la relativización de la importancia de los determinantes externos del cambio social, motivando un viraje que centra los estudios de gobernanza educacional en la incidencia de las dinámicas relacionales y la construcción de significados de los sujetos (CORBETTA, 2009).

\section{El proceso de descentralización en Chile: revisión del tema}

Las reformas de la administración pública implementadas desde los 90' en Latinoamérica, planteaban medidas para hacer más eficiente al Estado, como respuesta a los cambios que le antecedieron, centradas en la reducción de su tamaño y de la potestad del mismo. En este caso se proponían aumentar la eficiencia y capacidad del respuesta del Estado mediante la descentralización territorial político-administrativa (HOXBY, 2002; MARCEL, 2002, MOE, 2001).

\section{El debate sobre descentralización}

El análisis del proceso de descentralización impulsado en Chile reviste particularidades, al tenor de los planteamientos de Eaton (2004) y Mardones (2006), se formulan dos hipótesis para explicar su devenir. Primero, la descentralización es resultado del proceso de democratización, y segundo, ocurre por consideraciones electorales. Ambas cuestiones son claves: respecto de la democratización, la descentralización en Chile tuvo 
claro sentido restaurador de la política local, como práctica antigua del país, descontando el período dictatorial de Pinochet. Por su parte, la reforma regional y la instalación de su institucionalidad, son el efecto no premeditado de esta restauración, bajo un sello electoral derivado de las transacciones políticas que siguieron a la derrota del dictador en1988 y las negociaciones con el gobierno que culminan en el plebiscito de 1989, dirigido a cambiar cuestiones claves de la controversial Constitución Política de 1980, para asegurar cambios políticos intransables del entonces bloque opositor triunfador del plebiscito y también, como garantía de la derecha derrotada para "no desmantelar" la constitución.

En este marco, Chile ha privilegiado las políticas centralistas, favorecida por una escasa presión del poder local por mayor autonomía, evidenciando el conflicto político que subyace

Lo que es particularmente llamativo es que la descentralización va en contra de los intereses fundamentales de los actores políticos dominantes en el país, con sede en Santiago, entre ellos los políticos y líderes de partidos, legisladores nacionales y los poderosos burócratas. La hostilidad de estos actores hacia la descentralización, combinada con la debilidad de los actores subnacionales en el sistema político de Chile, nos Ilevan a no esperar mucha descentralización. No obstante, se produjeron cambios importantes (EATON, 2004: 220).

El desarrollo de los sucesos desde 1990 requiere para su comprensión dejar a firme que el régimen dictatorial instala cierta institucionalidad subnacional, la que posteriormente se refuerza producto de los arreglos políticos de la nueva democracia "... el régimen de Pinochet representa un momento crítico, en primer lugar porque pone en marcha e instala fuertes instituciones subnacionales, y segundo porque pone en movimiento más cambios descentralizadores, incluso después de que él se retiró del poder" (EATON, 2004: 236), ello se evidencia no solamente porque se producen algunas transformaciones post-cambio de gobierno, sino que en tanto la democracia se distancia de su hito clave (1990), más lento se hacían estos procesos de reforma, pues las iniciativas quedaban estancadas en el parlamento 5 .

No obstante la débil convicción por la descentralización de las autoridades políticas, salvo excepciones, no es menos cierto que la política de la descentralización es un tema relevante tanto para el debate público como para el estudio especializado. Con respecto a la discusión nacional, esta ha priorizado los aspectos técnicos, haciendo abstracción de la política. Definida la descentralización como una transferencia de poder político, dineros fiscales y/o atribuciones administrativas a gobiernos subnacionales autónomos, se trata en esencia de una redistribución de recursos de unos actores hacia otros (MARDONES, 2006), siendo ese aspecto el elemento basal de la temática en análisis.

Los procesos de descentralización generan incertidumbre en el mediano y largo plazo. "Para los políticos del nivel nacional estas reformas son un 'negocio riesgoso'" (EATON, 2004c). Adicionalmente, se han evidenciado dos efectos no previstos de mediano y largo plazo: potenciar el poder político de los actores municipales en relación al gobierno central, lo que, a su vez, les ha permitido presionar por más descentralización; y segundo, la reforma regional ha posibilitado una demanda más reciente por la elección directa de los consejeros regionales, así como la elección de un presidente regional (MARDONES, 2006).

Aunque hay una resistencia política a estos cambios descentralizadores, sería imposible de entender hasta qué punto el debate se ha desarrollado en Chile, sin hacer referencia a la labor de la SUBDERE. (EATON, 2004: 237). Confirmando con este proceder, según Mardones (2006), la hipótesis del mismo "Eaton (2004c:18) quien señala que habiendo presiones de 'abajo', la descentralización es resultado de conflictos e intereses divergentes entre los actores de 'arriba'”. Igualmente, las reformas alteran la distribución inicial de recursos, cambian la correlación de fuerzas y presionan por reformas adicionales. Ello se evidencia por la poca fortaleza que presentan los actores locales y regionales, quienes, salvo excepciones, han tenido un papel de menor importancia, "Dada la debilidad relativa de los actores subnacionales, si en Chile se han aprobado reformas

5El caso emblemático es la elección directa del Presidente del Gobierno Regional y de los Consejeros respectivos, iniciativa que no tuvo avances importantes por 16 años hasta octubre de 2013, que por primera vez se eligieron en votación directa los Consejeros Regionales. 
descentralizadoras habría sido, porque los actores nacionales han capitalizado beneficios a partir de tales reformas" (EATON, 2004b: 87), aunque producto de ellas hay casos señeros de posicionamiento de actores locales (esencialmente Alcaldes) hacia planos de relevancia regional o nacional, cuestión que se relaciona con las estructuras de poder instaladas más que con eficiencia de los actores para instalar sus demandas (MARDONES, 2006).

\section{Descentralización educacional: contexto político-social}

Los insatisfactorios e inequitativos resultados educativos se asocian con las tensiones en el sistema educacional instaladas desde los 80' por las sucesivas reformas educacionales entre dos modelos de administración de bienes públicos, claramente divergentes: el modelo neoliberal de Estado Subsidiario y el Estado Benefactor (DONOSO, 2013; OCDE, 2004). Tras la privatización de una parte fundamental de la educación primaria, secundaria y terciaria, el Gobierno no ofrece regulaciones suficientes que resguarden simultáneamente la libre competencia con el acceso a una educación de calidad para todos, ni siquiera con la nueva Ley General de Educación (2009) (EYZAGUIRRE, MARCEL y TOKMAN, 2005; DONOSO, 2013; ATRIA, 2014), siendo este factor un catalizador de los conflictos que se manifiestan en la educación desde el 2011 en adelante.

Las reformas liberales descentralizadoras de los 80' restringieron el mandato del Ministerio de Educación para intervenir sobre la gestión de los responsables escolares públicos y privados, además disocian la institucionalidad pública del sistema educacional, quedando la responsabilidad de los resultados educativos en "tierra de nadie", pues el Ministerio carece de recursos legales y financieros para operar en esta materia. El diseño organizacional del sistema educativo asigna funciones discretas a sus distintos componentes sin que tengan una de injerencia real en las distintas etapas del proceso educacional (COX, 2003). Las funciones de supervisión técnico pedagógicas están asignadas al Ministerio de Educación; la administración de los recursos a los Departamentos de Administración Municipal de Educación y la conducción del proceso educativo a los establecimientos escolares (GALLEGO; SEEBACH, 2007:79; OCDE, 2004; DONOSO; ARIAS, 2011).

La disociación ideológica representada por los dos modelos sociopolíticos (y económicos) en tensión, y la disociación institucionalizada de funciones del sistema educativo se amplifican en el marco centralizado en el que operan. El Estado intenta correcciones que van desde instrumentos de planificación educacional regional, programas y políticas de mejoramiento educacional hasta proyectos de ley, sin alcanzar los estándares de la OCDE ni satisfacer los requerimientos gremiales ni las demandas de los estudiantes y sus familias, como lo muestran los conflictos del 2006 y 2011.

Las limitaciones del sistema educativo se muestran también en la dificultad del gobierno central para buscar soluciones participativas a un problema eminentemente público, que convoca a todos los sectores del gobierno. La alta varianza en la matrícula y en los recursos financieros de que disponen son muy heterogéneos en el sistema comunal de educación pública, dando cuenta de una realidad que el nivel central tienden a omitir ${ }^{6}$ (MONTT, 2006; DONOSO; ARIAS, 2011, 2013). El traspaso de los servicios educacionales a los municipios, realizado unilateralmente por la Dictadura y mantenido por la Concertación, aumenta la inequidad social en las oportunidades a una educación de calidad, por cuanto se trató de un cambio institucional, normativo y financiero, características que perduran con bemoles menores hasta el presente (DONOSO, 2013).

El municipio sigue siendo el principal proveedor de educación pública, con algo menos del 40\% de la matrícula nacional, este proceso de "pérdida de estudiantes" que se ha registrado con fuerza desde los años 90', atiende en forma a los sectores vulnerables de la población, a la par que aumenta la desigualdad social en el país (EYZAGUIRRE et al., 2005:43; RACZYNSKI; SALINAS, 2006; HSIEH; URQUIOLA, 2002, 2006;

6Hay 345 municipios en el país, sus sistemas educativos van desde menos de 100 estudiantes hasta más de 40 mil. Desde 1 establecimiento hasta cerca de 100. Con una serie de diferenciaciones muy importantes, Los 150 municipios más pequeños tienen menos de 2000 estudiantes. 
MIZALA; TORCHE, 2012). El 46\% (1,8 millones de estudiantes) de niños y jóvenes, que corresponden al 70\% de la matrícula escolar nacional que asiste a escuelas públicas proviene de hogares de escasos recursos (CONSEJO ASESOR, 2006). Los resultados de aprendizaje de la educación municipal en las comunas más pobres y pequeñas, asociadas generalmente al ámbito rural o semiurbano, están por debajo del promedio nacional, disminuyendo más rápidamente su matrícula y enfrentando importantes problemas de gestión y financiamiento (DI GROPELLO, 1999; GARCÍA-HUIDOBRO; BELLEI 2003; MARCEL; RACZYNSKI, 2009; DONOSO; ARIAS, 2011, 2013).

\section{Contexto socio-educacional de la Región del Maule (Chile)}

La ruralidad y pobreza de la región del Maule $^{7}$, son condiciones estructurales que determinan históricamente la dinámica de los sistemas educacionales y la baja calidad de los resultados de la educación. La precaria inserción laboral de la población, la estacionalidad de la industria agrícola y forestal, contribuyen a situarla como la segunda región más pobre y desigual del país, donde las personas más ricas obtienen ingresos autónomos 11,3 veces superiores que las más pobres (GOBIERNO REGIONAL, 2009:15). La región, a la par del país, ha logrado disminuir sostenidamente la pobreza, aunque aumenta consecutivamente la brecha con otras regiones. Estas condiciones dificultan su adecuación a las políticas y programas de mejoramiento educacional estandarizadas y diseñadas con una visión de ciudad grande y de territorio urbano.

El rezago regional respecto del país se replica en el ámbito educacional. De los 518 establecimientos de educación pública emplazados en la región, el 65\% son rurales. El Maule, si bien logra superar su posición decimosegunda o antepenúltima en la escala de medición de la calidad de la educación, pasando al sexto lugar en esta dimensión, se mantiene muy por debajo del promedio nacional; destacando los altos porcentajes de analfabetismo en los que duplica la tasa nacional (GOBIERNO REGIONAL, 2009:15). A la precariedad del capital económico se suma el cultural. Se trata de una región con un fuerte componente rural, con algo menos del 40\% de la población, donde los procesos de descampesinización, asociado a la migración interregional, determinan un envejecimiento acelerado de la población en comparación al resto del país (GOBIERNO REGIONAL, 2009). Consecuentemente, la matrícula escolar de los sistemas educacionales más pequeños, afectos a la condición de ruralidad, se reduce en forma acelerada a raíz de lo señalado. Concomitantemente, la dispersión geográfica y extensión territorial de algunas comunas condiciona un aislamiento relativo en comparación con las ciudades cabeceras de provincias y de regiones, lo que desincentiva la inserción laboral de aquellos docentes más calificados y eleva los costos de operación (DONOSO; ARIAS, 2011, 2013). Pese a la merma de matrícula, rigidez ${ }^{8}$ del Estatuto Docente y la creciente escasez de empleos regulares en el sector educación exacerban el carácter clientelar de las contrataciones.

En síntesis, los sistemas comunales de educación del Maule presentan severas dificultades para regular las tensiones del sistema educativo dado su pequeño tamaño, menores recursos y costos más elevados, y su precariedad en materia de institucionalidad y capacidad de operación. La descentralización educacional impulsada en los años en análisis, no ha logrado revertir los problemas de inequidad para el acceso y permanencia de los estudiantes en un sistema que provea una educación de calidad, esencialmente porque los sistemas comunales de educación pública reflejan la elevada inequidad del país, reuniendo de forma preferente a la población más vulnerable, incidiendo en que sus costos de operación, equipamiento e infraestructura sean cubiertos parcialmente por los recursos públicos centrales, debiendo a nivel subnacional - esencialmente local-

7 El Estado chileno es unitario, el Presidente es representado por el Intendente en cada región, solamente los Alcaldes son electos democráticamente.

8El Estatuto Docente dictado en 1991 con sucesivas correcciones fue motivado por el desamparo de los docentes del sector público ante la autoridad (el Alcalde), con el paso del tiempo se ha flexibilizado su articulado, no obstante -a juico de algunos- protege en grado elevado a los docentes, siendo considerado necesaria su reforma, aunque los términos de la misma varíen según los actores involucrados. 
hacer inversiones per cápita/ alumno comparativamente mayores que las unidades territoriales de mayor tamaño poblacional, lo que incide en la calidad del servicio educativo. Estos sistemas requieren para su desarrollo de propuestas específicas, territorialmente integradas, no obstante una serie de dinámicas rebasan al ámbito educativo dificultan su diseño.

\section{La toma decisiones en educación el gobierno del Maule}

El desarrollo regional implica tener recursos, implica tener una coherencia política, implica tener una estrategia, implica tener una decisión regional que uno le diga al centro: "Oiga, pero yo quiero esto y no lo que usted está proponiendo" [Participante № 10, funcionario Gobierno Regional del Maule 2009].

El principal resultado del estudio es que pese a los avances del país en materia de descentralización y dada la gravitación de los bajos logros del sector educacional en la región, no se registra una acción educacional subnacional concordada y eficaz en esta materia entre el gobierno regional y los gobiernos locales, ni tampoco se revela una necesidad sentida y demandante de los principales actores políticos del gobierno subnacional por crear un proyecto educativo con características distintivas que responda a las necesidades específicas del territorio.

De igual forma, los partidos políticos no disponen de propuestas educativas subnacionales, el gobierno regional sigue los lineamientos del gobierno central en esta materia. De haber un proyecto educativo territorialmente orientado, éste no se encuentra formalizado, ni reconocido como tal en las instancias del gobierno subnacional. Aspectos concordantes con lo expresado en los textos de Eaton (2004) y Mardones (2006), en que las demandas por reformas provienen más de los actores centrales y no de los gobiernos subnacionales, siendo frecuente que no existan proyectos propios dirigidos desde los gobiernos subnacionales. El Maule no es la excepción.

De todas formas, se observa la tendencia, en las propuestas de los actores consultados, a valorar el territorio como contexto sociocultural, es decir como plataforma de integración económica de la región hacia el mundo globalizado. Entre los consejeros regionales existe una visión dominante en pensar el eventual proyecto educativo de la región como herramienta para formar el capital humano requerido, según un plan de desarrollo productivo centrado en unidades territoriales que agrupen, desde una lógica de economía de escala, un conjunto de pequeños sistemas educativos. Otro aspecto que se observan a partir de los actores, es el tránsito desde las demandas de educabilidad hacia aquellas, técnico pedagógicas y cualitativas expresadas en el imaginario colectivo, a saber "ir más allá de los ladrillos de la sala de clases" o "abrir la caja negra de lo que ocurre en el aula". Se plantea la importancia del seguimiento y evaluación de los programas y políticas educativas.

Un grupo minoritario de funcionarios municipales se proyecta hacia una educación para la formación ciudadana, opuesta a las tendencias tecnologizantes y a la competitividad económica que dominan el panorama. Esta mirada emerge desde una visión diacrónica y crítica, que resalta la pérdida de la dimensión deontológica en la formación docente y en la práctica educativa. También los funcionarios municipales se sienten particularmente interpelados por la necesidad de recuperar para la educación la identidad rural, de la cual tanto las políticas educativas como ellos mismos se encuentran distantes. Expresan con nostalgia y culpabilidad como lo rural "esta a la vuelta de la esquina" en sus comunas, pero a la vez invisibilizado en las políticas educativas.

El análisis de las condiciones asociadas a la escasa reflexión en el gobierno subnacional sobre el proyecto socioeducacional, se explica en parte debido al feble proceso de descentralización que deprime el potencial generador y articulador de políticas educacionales. En efecto, la normativa vigente restringe las facultades de los gobiernos subnacionales para dirigir el desarrollo regional local, por lo que su empleo depende de la voluntad de los actores políticos y de los respectivos ministerios involucrados (SUBDERE, 2001). Incluso el Gobierno Regional como máximo organismo descentralizado territorialmente, investido de facultades normativas, resolutivas y fiscalizadoras, y que es responsable de elaborar la Estrategia esta limitado en estas funciones y depende ampliamente del Gobierno Central (Art. 28, DFL 19.175 Gobierno de Chile, Ley 19.175). La 
ley no le entrega atribuciones que resguarden jurídicamente su intervención en asuntos sectoriales, incluida la educación, cuya autoridad es exclusiva de las autoridades del Gobierno Central. El Consejo Regional (CORE), principal organismo representativo de la ciudadanía regional, cuya finalidad es "hacer efectiva la participación de la comunidad regional" y está "investido de facultades normativas, resolutivas y fiscalizadoras" (Art. 28, DFL 19.175), limita su accionar a priorizar las inversiones presentada por el Intendente quien preside y veta en esta instancia la cartera de proyectos de inversión, en su calidad de Jefe del Gobierno Regional.

En este contexto de restricciones jurídicas e institucionales, las autoridades locales desestiman los instrumentos de planificación, que son dispositivos estratégicos para la modernización del Estado y representan la voluntad política desde el gobierno central de dotar de autonomía a las regiones. Los funcionaros municipales ven coartadas las posibilidades de incidir en las decisiones de los sistemas educacionales comunales a través de propuestas programáticas, ante la expectativa de encontrar resistencia en alcaldes y Concejos Municipales, al presentar iniciativas distintas. De hecho, definen las herramientas de planificación como: (i) Dispositivos de control administrativo financiero y laboral del Gobierno Central desprovistos de incentivos; (ii) Postulados teóricos interesantes, pero disociados de la realidad local; (iii) Desafíos técnicos frente a los cuales no se sienten necesariamente competentes; (iv) Instancias que objetivan su rol de operarios ya que no se les considera en su diseño; (v) Elementos disociados entre sí, cuyas implicancias para el desarrollo comunal es ambigua ya que no son evaluados ni tienen poder jurídico institucional vinculante; (vii) Instrumentos carentes de seguimiento, rigurosidad y sistematicidad lo que amplifica la brecha entre lo planificado y lo que efectivamente se hace. Se plantea que "la mayoría de las veces no se respeta el instrumento".

Adicionalmente, la Estrategia de Desarrollo Regional ${ }^{9}$ (la estrategia) que representa la principal posibilidad de aportar sinérgicamente desde lo público con un enfoque territorial integrado al desarrollo regional, se la denota como: un amplio y estandarizado discurso teórico especializado, basado en diagnósticos tecnocráticos, no acabados, con escaso alcance operativo. Es recurrente entre los actores considerar que los instrumentos de planificación no aportan elementos sustantivos y proactivos para el desarrollo del territorio:

Está bien la Estrategia, pero ahora, aterricémosla. Bajémosla, bajémosla, bajémosla. Es decir, en cada tema que toca la Estrategia bajemos a la letra chica. Agreguemos otra etapa más de la Estrategia. Necesitamos Ilegar un poco más al terreno mismo: Cómo un alcalde con sus recursos, cómo un jefe de servicio con sus recursos, puede implementarla en forma fácil. Más amigable. Hoy día la Estrategia no es amigable. [Participante № 53, funcionario Gobierno Regional 2009].

La "Estrategia" es un reto para los consejeros regionales: les demanda conocimientos políticos y competencias técnicas para aportar a su diseño. No obstante "los gobiernos regionales no cuentan con una estructura profesional estrictamente encargada de manera sistemática para estudiar, implementar y evaluar las iniciativas de desarrollo regional" [Participante №10, funcionario Gobierno Regional 2009]. Otra limitante de los instrumentos de planificación regional es su dispersión representada en múltiples, discretos y pequeños proyectos comunales y sectoriales. En la región del Maule, sólo durante el año 2009 el Fondo de Desarrollo Regional, instrumento de inversión que sigue los lineamientos de la Estrategia, financió 933 iniciativas, de ellas el 17\% fueron proyectos de envergadura regional de infraestructura (salud, educación y servicios básicos y empleo), el grueso de la inversión, el 83\%, se tradujo en micro proyectos comunales, mayoritariamente de deporte y cultura (GOBIERNO REGIONAL, 2009).

Dispersión similar ocurre en la planificación educacional donde el PADEM ${ }^{10}$ (Plan Anual de Desarrollo Educativo Municipal) y el PLADECO (Plan de Desarrollo Comunal), privilegian inversiones en obras de infraestructura

9 Esta Estrategia provee una "imagen futura de región" siendo un eferente para evaluar la pertinencia de las inversiones de decisión regional (SUBDERE, 2011).

10 El objetivo del PADEM es la planificación y evaluación participativa anual de las acciones de los sistemas educativos municipales en articulación con IOS PLADECO. EI PADEM requiere un diagnóstico y proyección de la evolución de los distintos aspectos del sistema educativo comunal que orienten las decisiones educacionales (Ley $\mathrm{N}^{0}$ 19.410/1995, artículos $4^{0}$ a $6^{\circ}$ ). 
educativa no articuladas y postergan un abordaje conjunto (sistémico) que recoja la especificidad de la problemática regional.

Lo que pasa con el PLADECO es que se llenan de 5.000 problemas y no saben priorizar cuáles son los esenciales, lo que es desarrollo integral. Andan preocupados de la perilla chica, como se dice y de la formulación de planes chicos. Entonces falta el desarrollo integral del PLADECO, una cuestión más grande. Entender que ellos son el cerebro del desarrollo de la comuna. Pero si tú ves, los PLADECO son puros pedidos de cuestiones chicas. Tengo mis dudas si se apegan a la Estrategia de Desarrollo Regional [Participante $\mathrm{N}^{\circ} 56$, funcionario municipal 2009].

Otra dimensión es la "mentalidad funcionaria" que acota las soluciones al contexto comunal o micro comunal, argumentando que los instrumentos de planificación comunal "están hechos para satisfacción local":

[El entrevistado emite su opinión sobre la Estrategia] Nosotros no trabajamos para un tema regional, nosotros trabajamos para un tema local; que la suma de todo lo local o comunal luego lo dialogaran a nivel regional, pero nosotros trabajamos por un tema Iocal. Nuestro PADEM [Plan de Desarrollo Municipal] está orientado a planificar acciones locales, en las escuelas, pero nosotros no estamos que vayan a tener un impacto regional, por ningún motivo. Nosotros trabajamos para lo Local ... el DAEM [Departamento de Educación Municipal] planifica para lo interno que impacta la Gestión de la Administración Local [Participante el Nº5, funcionario municipal].

En la práctica, las herramientas de programación se emplean en la resolución focalizada de las urgencias presupuestarias comunales, situación que se registra también en otras regiones (DELPIANO, 2003:21-27), respondiendo al mandato de equilibrio territorial de los gobiernos subnacionales (SUBDERE, 2001). En otros casos representan, según la evidencia del estudio, repeticionesde documentos anteriores:

Queremos que la Estrategia sea distinta al marco general que se va a dar a conocer en otras regiones. Si uno ve la Estrategia que nos presentaron, no es tan disímil a lo que puede ser en la décima región, o en la cuarta porque son marcos generales. Es casi una copia de otros lados. [Participante N 56, funcionario Gobierno Regional 2009].

Los instrumentos de planificación no han tenido la consistencia necesaria y suficiente. Hay un PADEM, pero generalmente de uno a otro, solo cambia la forma pero no el fondo. El contenido es el mismo, no se define políticas de largo plazo [Participante $\mathrm{N}^{\circ} 54$, funcionario municipal 2009].

Yo pienso que al final, todas las actividades que realiza el Gobierno Regional y el municipio pueden alinearse con la Estrategia [Participante $N^{\circ}$ 2, funcionario Gobierno Regional].

Las dificultades de la región para elaborar propuestas educacionales específicas e innovadoras se ratifica en las tendencias de inversión. El gasto público social en Educación (PLADECO) sigue las orientaciones de las políticas nacionales, privilegiando cobertura y permanencia escolar, principalmente en pre básica, como también la educación técnico-profesional y el incremento de la Jornada Escolar Completa (GOBIERNO REGIONAL, 2009; SUBDERE, 2011). Igualmente, la planificación educacional se expresa en objetivos, metas e indicadores nacionales de rendimiento escolar, cobertura, retención y acceso escolar (GOBIERNO REGIONAL, 2009). Entre los escasos ámbitos que han sido abordados con éxito en planificación educativa es la infraestructura y cursos de perfeccionamiento para profesores requeridas por la Ley el Sistema de Subvención Preferencial y los Planes de Mejoramiento de la Educacional Municipal por los Departamentos de Educación Municipal. No obstante, se excluyen importantes temas técnico pedagógicos (OCDE, 2010:25) y se desconoce si estos instrumentos resuelven la falta de planificación a mediano plazo que afecta a los municipios, ya que su integración al tema es marginal y se requiere voluntad política para hacerlos funcionales (ESPÍNOLA; SILVA, 2009; ORTEGA el at., 2009; OECD, 2009).

Los problemas de los instrumentos de planificación traspasan el ámbito conceptual/técnico de su misión y diseño, situándose en el contexto en que operan y en la forma que son interpretados e implementados por los actores. Es decir, son restringidos por la debilidad del respaldo intersectorial y de los sistemas de seguimiento que garantizan el cumplimiento de la política de descentralización educativa expresada por ley (GOBIERNO DE CHILE, 2002; CASTRO, 2010; GONZÁLEZ, 2005). Estos dispositivos lejos de corregir las asimetrías de poder, 
conocimientos y competencias, entre centro y regiones, actúan como reproductores del poder del Gobierno Central, asimilándose a la lógica burocrática administrativa sin poder vinculante con la realidad regional, contrariamente al rol protagónico y gerencial que se le atribuye desde el discurso de la modernización.

Para hacerles eficientes es necesario transformar el marco jurídico institucional y sus procesos de generación y gestión. No obstante, hay una falta de comprensión de la proyección política de la educación en el marco de la identidad territorial que no se resuelve con una normativa, implicando la concepción de una política regional como la simple suma de cada política local; y además, la escasa capacidad del gobierno regional para crear políticas regionales integradas, articulando los recursos del FNDR y otros fondos sectoriales entregados en forma directa a los municipios, y que de haber existido acciones sinérgicas sus impactos habrían sido muy superiores al respecto, ejemplo los fondos PMGM (o FAGEM) de educación principalmente de los años 2008/9 y 2011/12/13.

En síntesis, los resultados muestran dos aspectos centrales: primero, que conforme se han presentado las dinámicas de implementación de las políticas de descentralización, sus diseños responden a "iniciativas centralizadas de descentralización", con un aporte menor sino marginal de los actores subnacionales. Proceso marcado por una institucionalidad débil, por actores locales que han alcanzado mayor peso que los regionales, por iniciativas impuestas desde el centro sin una contraparte que haga valer las particularidades del contexto, ante lo cual las políticas subnacionales representan adecuaciones que sortean las tensiones entre instrumentos diseñados para diversos fines, con la visión de las políticas de descentralizadoras desde perspectivas incluso disonantes. Cuestión que suele omitirse a la hora de hacer efectiva estas materias. La toma de decisiones en el plano en análisis no se rige por proyectos subnacionales, sino por la "bajada de los proyectos nacionales hacia lo subnacional".

\section{Gobernanza y descentralización}

"... el otro día el Intendente nos planteaba [Consejeros Regionales] ¿Qué queremos nosotros como región? Y ése es el tema que yo creo que está pendiente todavía, una conversación con todas las instancias políticas y privadas también [Participante $\mathrm{N}^{\circ} 25$, funcionario Gobierno Regional 2009].

Un argumento para comprender el vacío de iniciativas educativas territoriales es la falta de discusión de políticas estratégicas en los gobiernos subnacionales respecto de las vías de desarrollo, asociada esta condición a la dominancia de las lógicas de reproducción del poder partidistas que orientan las decisiones de inversión regional que distan de plantear propuestas en este sentido, respondiendo al centralismo político partidista de Chile, salvo excepciones. Esto conduce a problemas de gobernanza, entendida como el estilo de gestión que se objetiva en el conjunto de bienes, normas y procedimientos que componen su sistema organizacional. La gobernanza está configurada tanto por las tendencias políticas nacionales como por la organización del poder en los gobiernos subnacionales. Respecto a este último, su análisis considera dos factores: La influencia de la lógica partidista que orienta las prácticas decisionales de los partidos políticos que participan en el gobierno regional, que desenfoca a los actores de un proyecto de desarrollo regional territorialmente; y la débil vinculación de los Gobiernos Subnacionales y partidos políticos con la sociedad civil ${ }^{11}$.

Respecto de la incidencia de las dinámicas partidistas en las posibilidades de desarrollo regional, el punto central es que el accionar de los partidos políticos, entendidos como representantes de propuestas de cambio social e ideario pro regionalización, deprimen los intentos de cambio. Salvo orientaciones básicas sobre la regionalización, que forman parte de las declaraciones de principios de los partidos, éstos no cuentan con propuestas formales de desarrollo educacional para los niveles subnacionales. De allí el diagnóstico compartido por muchos consejeros regionales: "hoy tenemos los recursos pero falta la discusión". La discusión política sobre el devenir de la región es asistemática, generalmente no traspasa las prácticas de negociación político

11La sociedad civil, igualmente, es débil en estos territorios salvo excepciones. 
partidista del Gobierno Subnacional. Además, la sociedad civil en las localidades intermedias y menores está escasamente empoderada.

La alineación de los poderes locales con inversiones de mayor envergadura requiere superar aquellas prácticas que garanticen la distribución del poder sobre las decisiones de inversión pública. Ello debido a que proyectos territoriales amplios, disputan los espacios de influencia local/electoral a las autoridades, al sobrepasar sus ámbitos de competencias territoriales. Es decir: tensionan el modus operandi de las elites nacionales, ordenada en partidos políticos cuya estructura se superpone y confunde con la del Gobierno subnacional.

Lo del deporte fue grosero: para no crearse problema el CORE [Consejo Regional] a puertas cerradas, con el Intendente, se distribuyeron mil millones de pesos entre 19 consejeros regionales. Entonces tú dices; yo tengo 55 millones en mi bolsillo. Entonces, voy donde mi amigo del deporte a la Junta de Vecinos, o del club deportivo y le digo: hazme un proyecto por tres millones. Porque va a salir compadre, porque es mi plata. Entonces todos hicieron eso. Juntaron los proyectos hasta 55 [millones], de 10 de 50100 de un millón. Los otros 1.500 proyectos de gente que participó en un proceso transparente, que no llamó nunca a un Consejero, que no se reunió en lobby, ahí están. Se les fue. Asqueroso, pero así es. Es la realidad. Esas son las prácticas. Porque además estamos en un período eleccionario es asunto de rentabilidad política, de voto. [Participante $N^{\circ} 54$, funcionario Gobierno Regional].

La "lógica de reparto" funciona para mantener las políticas de alianzas entre partidos políticos (FERNÁNDEZ, 2009) no obstante, aplicada a la distribución de fondos regionales de inversión, condiciona y limita los espacios de autonomía de las autoridades regionales, siendo contraproducente con la permeabilidad del Gobierno Subnacional hacia nuevas iniciativas de desarrollo. Un proyecto regional consensuado implica reasignación del poder regional, exigiendo a las elites hacer prevalecer el desarrollo regional a estas lógicas de reproducción del poder político. En definitivo, de la voluntad de los partidos políticos dependerá mucho del ritmo de la descentralización pensada desde los gobiernos regionales (VALENZUELA, 2007).

La auto-referencialidad de las prácticas de gobernanza implica otro obstáculo: la falta de asidero de las políticas de mejoramiento de la educación en las demandas ciudadanas. La orientación partidista descentra a los principales actores responsables de la generación de políticas regionales vinculantes con la ciudadanía. Una propuesta de desarrollo regional con proyecciones requiere nutrirse de la diversas miradas y realidades de la región, convocando a los gobiernos locales y a la sociedad civil. No obstante, la búsqueda electoral actúa en sentido inverso, no sólo frustra a la sociedad civil, sino que incita al Gobierno Subnacional a parcelar los proyectos de desarrollo, priorizándose múltiples microproyectos dirigidos, principalmente, a los sectores urbanos más densamente poblados de la región. Esta práctica de "centralismo regional", reproduce la lógica nacional a escala territorial, profundizando la desigualdad de acceso a los recursos de los sectores de menor densidad poblacional, desventajados por la distancia geográfico/cultural para acceder a las redes del gobierno. Por su parte, la sociedad civil, que tiene un importante poder de presión hacia las colectividades políticas, suele desconocer el rol de los consejeros regionales y no es un elemento catalizador en este sentido.

En síntesis, las dinámicas centralistas nacionales y regionales de reproducción y equilibrio del poder político que dominan en el Gobierno Subnacional, minan las posibilidades de abocarse a proyectos estratégicos de desarrollo regional, favoreciendo la fragmentación de los fondos de inversión y las miradas de corto plazo sobre el tema. Los instrumentos de planificación regional se insertan en esta dinámica y no logran reformar las prácticas tradicionales de gobernanza centralizada entre los distintos niveles de Gobierno y los actores que operan a nivel central (OECD, 2009; FERNÁNDEZ, 2009), ni tampoco cumplen con generar políticas regionales territoriales según se les encomienda (GOBIERNO DE CHILE, 2000).

Los partidos políticos privilegian prácticas que reafirman a las elites del Gobierno Subnacional, reproduciendo "regionalmente" el centralismo en esta escala. El distanciamiento progresivo de los partidos políticos con la ciudadanía, asociado a la crisis de representatividad de las colectividades, al desconocimiento históricos de estas materias y cierta pasividad de la sociedad civil, favorecen este proceso, pues no ejercen presión significativa sobre el Gobierno Subnacional en pos de demandas territoriales integradas. En definitiva, pese al 
aumento de recursos de inversión y los avances en gestión, se ha producido un vacío en la discusión prospectiva que sostenga el proceso de regionalización. Ni el Gobierno Regional, los partidos políticos, ni la sociedad civil asumen un liderazgo, por tanto ello no ha estado en sus prácticas habituales, y el gobierno subnacional no ha sostenido una visión sistémica que facilite esta labor, permaneciendo al arbitrio de las políticas del centro, dada la fuerte asimetría de capacidades y poder entre el centro y los gobiernos subnacionales, brecha cuya magnitud es un factor gravitante para que los gobiernos regionales no asuman un liderazgo de mayor proyección: se requiere de una política nacional de traspaso y generación de capacidades y empoderamiento de los gobiernos regionales, y en igual forma de los gobiernos locales, para impulsar una descentralización exitoso ${ }^{12}$.

\section{El Gobierno Subnacional del Maule, las políticas descentralizadas y la Educación}

El trabajo tuvo por fin analizar el desarrollo político de las instancias de gobierno subnacional en materia de toma de decisiones, referidas a las políticas que combinan elementos tanto nacionales con componentes subcentrales, abordándose en la política pública de educación, por relacionar en forma directa estos aspectos y por su creciente relevancia en las propuestas de desarrollo de los diversos territorios, bajo dos supuestos centrales: primero, las posibilidades de éxito de las políticas educacionales dependen del grado en que respondan a la realidad y necesidades definidas desde los propios actores que las piensan e implementan, implicando una propuesta de la sociedad liderada por los actores subnacionales, que sustente sus decisiones en políticas y programas educacionales legitimados bajo un horizonte consensuado de desarrollo regional, adquiriendo un potencial integrador al proveer contenidos sensibles y con sentido para los actores involucrados. Por ende el proceso de toma de decisiones tiene relevancia e impacto en el plano local y regional. El segundo supuesto es que se estima que una política educativa exitosa, es aquella que llega a todos los actores y niveles del sistema educativo, superando las oposición entre los componentes del sistema educacional, centro/región y región /comunas. Una alianza de este tipo debe estar mediatizada por un objeto consensuado que provea las bases para la acción educativa regional, es decir un proyecto de desarrollo educacional asentado en un ideal de desarrollo territorial de la región.

Este proyecto no solamente provee a las autoridades subnacionales de sustento para sus decisiones, sino que es un escudo de protección para desplazar el ámbito de estas decisiones, hacia nuevas atribuciones conquistadas al poder central, cuestión que en los hechos no es tal, como se desprende del análisis realizado, dado el bajo nivel de empoderamiento de autoridades subnacionales y de la ciudadanía: estos proyectos no son un instrumento de desarrollo, sino más bien mecanismos burocrático operativos de asignación de recursos y de toma de decisiones, que no representan un riesgo para las decisiones centralizadas.

El principal resultado señala que pese a los avances en descentralización y de la importancia que reviste la problemática educacional, no se ha logrado generar políticas educativas territorialmente integradas que respondan a las particularidades y demandas de los contextos comunales y regionales. En este marco, los instrumentos de planificación educacional local cumplen una función instrumental y se encuentran fuera de la lógica de acción de los gobiernos locales, debido a que las dinámicas políticas y administrativas superan los objetivos técnicos para los cuales fueron concebidos. Lo anterior en consecuencia de la precariedad de la descentralización a nivel subnacional, de la debilidad de su instrumentos, de la falta de discusión política en los Gobiernos Subnacionales sobre el desarrollo prospectivo de los territorios, cuyas decisiones de inversión se ordenan, esencialmente, según lógicas de reproducción del poder partidista o visiones centralistas acerca del desarrollo de esa región en particular.

Se constata, igualmente, que si bien el imaginario de los proyectos de desarrollo educacional reproduce las políticas centrales que priorizan el territorio como eje ordenador, se asume una visión más administrativa (en el

12 Mejorar la institucionalidad local es clave para el desarrollo del país. La mayor parte de las políticas públicas, especialmente las sociales se implementan en los municipios, el país tiene una gran atraso en este campo no solo en la Ley de Plantas Municipales, sino en la capacitación y calificación profesional de estos funcionarios. 
sentido convencional) que funcional de los territorios. Esta cuestión ocurre, porque la dinámica descentralizadora lo permite, pues los gobiernos subnacionales, en los hechos, no actúan como representantes de la ciudadanía ante el gobierno central, sino fundamentalmente al revés, representan el gobierno central ante la ciudadanía local. Es en el marco municipal (local) donde esta lógica no calza, pero la fuerte dependencia financiera de los municipios ante el gobierno central reduce los conflictos a tensiones menores, sin que se llegue a puntos de no retorno.

La dominancia de lógicas del poder político en el gobierno subnacional, es contraproducente con aquellas decisiones que requieren de proyectos consensuados y de largo plazo, como los educacionales, ya que se instrumentalizan las herramientas de planificación y se socava la discusión política sobre un proyecto educativo regional. De la misma manera, la mirada administrativo/burocrática comunal circunscribe la problemática educacional dentro de un territorio restringido y auto contenido que hace abstracción del potencial de desarrollo del horizonte regional o bien de propuestas asociativas con territorios equivalentes, ya que tampoco comprende dinámicas de acción intercomunal. Esta mirada microcomunal no es exclusiva de los gobiernos locales ya que atraviesa los distintos niveles político institucionales del Gobierno subnacional y del Gobierno central'13. El "partidismo" y la visión comunal como ejes articuladores de las decisiones de inversión educacional posponen la necesaria discusión sobre el desarrollo territorial integrado de la región y dan piso para que las decisiones adoptadas por las autoridades subnacionales no tengan un factor de contraste relevante, un baremo que evalúe su impacto en el mediano o largo plazo.

Se concluye, que se requiere de una ciudadanía y sociedad civil mayormente empoderada en los niveles subnacionales, cuestión sobre la cual aún falta mucho por hacer como país, pues implica una serie de condiciones que no están presentes en el medio subnacional "común", pasando por una ciudadanía demandante de sus derechos y cumplidora de sus deberes, tarea en la cual precisamente los sistemas educativos pueden hacer un aporte invaluable. Esto presionaría a que las autoridades - a su vez- exigiesen y sustentasen autonomía decisional para poder implementar políticas de descentralización cada vez más eficientes. El devenir de este proceso, a mediano plazo, implicaría en menos "partido" y más "proyecto políticos prospectivos" para construir un proyecto educacional que propenda al desarrollo territorial integrado, representativo de la sociedad civil y más ampliamente de la ciudadanía respectiva.

La falta de política y políticas deprime los espacios posibles de copar, que aún con limitaciones proveen las políticas descentralizadoras, especialmente en el campo de la educación, que podría demandar como fundamental esta condición, sin que la autoridad central pudiera negarse a tal argumento.

Paradojalmente subsiste, con presencia importante en las principales instancias políticas del Gobierno Subnacional, un ambiente despolitizado. Por lo mismo, y no solo asociado a la precariedad de la descentralización, los instrumentos de planificación se encuentran ampliamente deslegitimados en términos de su relevancia, aunque se cumplan los rituales que éstos demandan en su ciclo de gestión regular, y son objeto de prácticas conservadoras. Es decir, que son tratadas como meros formalismos que no logran revertir los vicios idiosincráticos, léase alcaldización, clientelismo y cuoteo político, a través de los cuales se perpetúan las elites regionales y locales. Los instrumentos de planificación significan para los actores regionales y comunales "contenedores vacíos" de sentido y de contenidos regionales.

En el caso propio del ámbito educativo subnacional, un sistema educativo sin proyecto educativo ideológica, cultural y territorialmente arraigado no es sustentable, ya que equivale a lo que Lechner (1990) señala como un proceso de modernización sin modernidad. Es decir, una estructura anacrónica y vacía de los contenidos significantes, que han hecho históricamente de la escuela un referente de socialización identitaria e integración social, función particularmente necesaria en el contexto de las enormes y crecientes desigualdades sociales y educacionales del país, e imprescindible para una nación que requiere construir un pacto social sólido, lo que implica proyectos educativos validados en esta perspectiva, con actores reconocidos y validos que les conduzcan.

13Los fondos de apoyo para la gestión comunal, implementados bajo distintos nombres desde el 2008 (PMGE- FAGEM) por el Ministerio de Educación son un ejemplo de la falta de políticas asociativas entre municipios para el potenciamiento de la educación pública de las comunas. 


\section{Referencias Bibliográficas}

ATRIA, F. Derechos sociales y educación: un nuevo paradigma de lo público. LOM Editores, Santiago de Chile, 2014.

BOISIER, S. "Crónica de una muerte frustrada: el territorio en la globalización, La recuperación de las políticas territoriales". Politika: Revista de Ciencias Sociales 1:11-25. 2005.

BOURDIEU, P. Raisons pratiques. Sur la théorie de l'action. Paris : Raisons d'agir. 1994

Science de la science et réflexivité. Paris: Raisons d'agir. 2001.

CARO, C. "Resultados SIMCE 2002, Derribando Mitos". Departamento de Estudios Municipales- División de Políticas y Estudios. Ministerio del Interior, Chile. 2005.

CASTRO, M. "Códigos para el Análisis de Política Educativa Local en Chile: Temas Pendientes". Ensaio18 (67):189-213. 2010.

CORBETTA, S. "Territorio y educación. La escuela desde un enfoque de territorio en políticas públicas". En De Relaciones, Actores y Territorios Hacia Nuevas Políticas para la Educación en América Latina, N. López y S. Corbetta (ed.), Buenos Aires, UNESCO, Instituto Nacional de Planeamiento de la Educación 263-275. 2009.

COX, C. "Las Políticas Educacionales de Chile en las últimas dos décadas del siglo". En Políticas Educacionales en el Cambio de Siglo.Cox, Cristián (ed.) Editorial Universitaria 19-114. 2003.

DONOSO, S. El Derecho a Educación en Chile. Nueva ciudadanía tras el ocaso neolibeal. Bravo y Allende Editores, Santiago, Chile, 2013.

; ARIAS, O. "Desplazamiento cotidiano de estudiantes entre comunas de Chile: evidencia y recomendaciones de política para la nueva institucionalidad de la educación pública". EURE, Vol. 39, N 116 enero, pp. 39 -75. 2013.

306). 2011.

"Diferencias de escala en los sistema de educación pública en Chile". Ensaio Vol.19, 71 (283-

DELPIANO, A. "Tareas y temas pendientes de los procesos de desarrollo regional y de descentralización". En Seminario A diez años de la creación de los gobiernos regionales, Evaluación y proyecciones. Ministerio del Interior, SUBDERE, Programa Universidades-Gobiernos Regionales, 21-27. 2003.

EATON, K. Designing Subnational Institutions: Regional and Municipal Reforms In Postauthoritarian Chile. Comparative Political Studies 37: 218. 2004. http:/cps.sagepub.com/content/37/2/218.refs.htmal. Revisado el 21 marzo de 2011.

ESPÍNOLA, V.; SILVA, M. E. "Competencias del sostenedor para una efectiva gestión del mejoramiento educativo en el nivel local: Una propuesta, Foco Educación". Santiago de Chile: Instituto de Políticas Públicas/ Expansiva UDP 3:1-34. 2009.

EYZAGUIRRE, N.; MARCEL, M.; RODRíGUEZ, J.; TOKMAN, M. "Hacia la Economía del Conocimiento: el camino para crecer con equidad en el largo plazo", Centro de Estudios Públicos 97:1-53. 2005.

FERNÁNDEZ, M. Institucionalidad Pública Desconcentrada y Gobernanza Territorial En Chile: Desafíos Para Un Desarrollo Territorial Equitativo, Santiago de Chile : FLACSO :1-21. 2009.

FUENTES, L.; ALLARD, P.; ORELLANA, A. "El Municipio y la gobernabilidad del territorio comunal. En La Reforma Municipal en la Mira Identificando los Municipios prioritarios en la Región Metropolitana: Complejidad comunal versus condiciones para la calidad de la gestión municipal”. Santiago de Chile: Expansiva 43: 159-208. 2007.

GALLEGO, F.; SEEBACH, C. "Indicadores de complejidad y resultado en el sector de educación municipal", En La Reforma Municipal en la Mira Identificando los Municipios prioritarios en la Región Metropolitana: Complejidad comunal versus condiciones para la calidad de la gestión municipal. Santiago de Chile: Expansiva, 159-208. 2007. 
GARCÍA-HUIDOBRO, J. E.; Cox, C. "La Reforma Educacional Chilena 1990-1998, Visión de Conjunto", En: García-Huidobro, Juan (ed) La Reforma Educacional Chilena.. Madrid: Editorial Popular 7-46. 1999.

GOBIERNO DE CHILE "Diagnóstico del proceso de descentralización en Chile". Chile, Ministerio del Interior, Subsecretaria de Desarrollo Regional y Administrativo, División de evaluación y cuentas públicas. 2000.

. "El Chile descentralizado que queremos: un proyecto de todos". Santiago de Chile: Subsecretaria de Desarrollo Regional y Administrativo. 2001.

"Texto actualizado / Diciembre 2009, Ley № 19,175, Orgánica Constitucional Sobre Gobierno y Administración Regional y Jurisprudencia Administrativa", Santiago de Chile: Ministerio del Interior, Subsecretaría de Desarrollo Regional y Administrativo Santiago: Gobierno de Chile. 2009.

. "SIMCE y educación", Santiago de Chile, Gobierno de Chile, Ministerio de Educación. 2009.

GOBIERNO REGIONAL DEL MAULE. "Anteproyecto Nacional de Inversiones". Talca: Gobierno de Chile. 2009.

GONZÁLEZ, P. "Estructura Institucional, recursos y gestión en el sistema escolar chileno". En Políticas Educacionales en el Cambio de Siglo La reforma del sistema escolar en Chile, editado por C. Cox 597 - 660. 2005

GROPELLO, E. "Los modelos de descentralización educativa en América Latina" Revista de la CEPAL 68:154170. 1999.

HOXBY, C. "Would School Choice Change the Teaching Profession?" The Journal of Human Resources 37: 846-891.

HSIEH, C-T; URQUIOLA, M. "The effects of generalized school choice on achievement and stratification: Evidence From Chile's Voucher Program”, Journal Of Public Economics 90:1477- 1503. 2006.

; __. "When school competes, how they compete? An Assessment of Chile's Nation wide school voucher program". Washington: World Bank's development group, 2002.

LECHNER, N. ¿Son Compatibles Modernidad y Modernización? El Desafío de la Democracia Latinoamericana?Santiago de Chile: FLACSO. 1990.

MARCEL, M.; RACZYNSKI, D. La Asignatura Pendiente. Claves para la revalidación de la Educación Pública de Gestión Local en Chile. Santiago de Chile: Colección CEPLAN, Uqbar. 2009.

"Las Opciones de Reforma del Estado". En Reforma de Estado, editado por S. Valdés. Dirección Pública y Compras Públicas. Santiago: Centro de Estudios Públicos II: 213-28. 2002.

MARDONES, R. "Descentralización y Transición en Chile". Revista de Ciencia Política, Vol. 26. № 1, 2006. 03 -24. www.scielo.scielophp?script=sci arteex\&pi. Consultado el 16 de marzo de 2011.

MIZALA, A.; TORCHE, F. Bringing the schools back in: the stratification of educational achievement in the Chilean voucher system. Journal of Educational Development. 32 (2012) 132-144

MOE, T. Vouchers, schools, and the American public". Washington: Brookings Institution. 2001.

MONTT, P. Hacia un sistema escolar descentralizado, sólido y fuerte. El diseño y las capacidades hacen la diferencia. Santiago Chile: Serie Bicentenario. 2006.

OCDE. "Informe de antecedentes del país para OCDE: evaluación de las políticas educacionales de Chile". Santiago de Chile: Organisation for Economic Cooperation and Development. 2003.

OCDE.Revisión de las políticas Nacionales de Educación. París: Organización para la Cooperación y el Desarrollo. 2004.

OCDE. Estudios Territoriales de la OCDE Chile. Santiago de Chile: Ministerio del Interior. 2009

OCDE. Revisión Económica Chile. Santiago de Chile. 2010.

ORTEGA, S.; NAVARRETE, J. Propuesta de Política Regional de Desarrollo Rural Región del Maule. Talca: Gobierno Regional del Maule. 2009.

PAVÉZ, M. A. "Municipios Efectivos en Educación". En Estudio de Caso 81, editado por MA. Pavéz, Santiago 
de Chile: Magister en Gestión y Políticas Públicas. Universidad de Chile, Facultad de Ciencias Físicas y Matemáticas, Ingeniería Industrial, CONICYT / Fundación Ford. 2004.

POLITEIA. "Estudio Mejoramiento de la Gestión y Calidad de la Educación Municipal". Informe Ejecutivo. Santiago de Chile: Gobierno de Chile/BID. 2008.

RACZYNSKI, D.; SALINAS, D. La Educación en el Nivel Local: ¿Cómo Manejar la Tensión Entre lo Técnico y lo Político? Santiago de Chile: Unicef, Ministerio de Educación-Gobierno de Chile. 2006.

SUBDERE. El Chile descentralizado que queremos. Subsecretaría de Desarrollo Regional, Ministerio del Interior, Santiago, Chile. 2001.

VALENZUELA, J. P. "Análisis Prospectivo De Las Capacidades Institucionales y de Gestión de los Gobiernos Subnacionales". Cono Sur Serie de Estudios Económicos y Sociales. Santiago de Chile: BID, Programa de Investigación en Educación. 2007.

ZEMELMAN, H. Horizontes de la Razón Historia y necesidad de utopía.Barcelona: Anthropos. 1992. 